ISSN 0103-9954

\title{
FAUNA DE FORMIGAS COMO FERRAMENTA PARA MONITORAMENTO DE ÁREA DE MINERAÇÃO REABILITADA NA ILHA DA MADEIRA, ITAGUAÍ, RJ
}

\author{
ANT FAUNA AS A TOOL FOR THE MONITORING OF A REHABILITED SAND MINE AT ILHA DA \\ MADEIRA, ITAGUAÍ, RJ
}

\author{
Marcos Paulo dos Santos Pereira ${ }^{1}$ Jarbas Marçal Queiroz ${ }^{2}$ \\ Ricardo Valcarcel $^{3}$ Antônio José Mayhé-Nunes ${ }^{4}$
}

\section{RESUMO}

A riqueza e diversidade de espécies de formigas podem ser maiores em ambientes de complexidade mais elevada devido a uma maior disponibilidade de nichos presentes. Em razão da conexão entre os organismos e as características dos habitats, as formigas têm sido utilizadas como ferramentas no monitoramento ambiental de áreas perturbadas. Neste estudo, foi investigada a estrutura da comunidade de formigas em parcelas utilizadas para reabilitação de uma área de mineração na Ilha da Madeira, Itaguaí, RJ. Encontrou-se uma fauna diferenciada de formigas e maior riqueza de espécies em parcelas nas quais foi utilizado um maior número de espécies arbóreas nativas do que em parcelas com poucas espécies arbóreas ou sem nenhuma intervenção conservacionista.

Palavras-chave: indicadores ambientais; comunidade de formigas; Formicidae; Baía de Sepetiba.

\section{ABSTRACT}

Ant species richness and diversity may be increased in more complex habitats because they provide more niches and diverse ways of exploiting the environmental resources. Because of the connection between the organisms and habitat characteristics, ants have been used as tools for environmental monitoring of disturbed areas. In this study the structure of the ant community was investigated in plots with different management strategies for the rehabilitation of a mined area at Ilha da Madeira, Itaguaí, RJ, Brazil. A different and richer ant fauna was found in plots with more native tree species than in plots with a low number of tree species or without conservationist management.

Keywords: environmental indicators; ant community; Formicidae; Sepetiba Bay.

\section{INTRODUÇÃO}

Os indicadores ambientais fornecem informações úteis para o monitoramento de práticas de manejo, visando à reabilitação de ecossistemas degradados (MAJER 1983/1992). Por serem muito abundantes e sensíveis a mudanças no ambiente, os insetos apresentam alto potencial para serem utilizados com esse propósito (ROSEMBERG et al., 1986). Entre os insetos, as formigas merecem destaque, pois, além das características citadas acima, são fáceis de coletar, relativamente fáceis de identificar e para as quais há um nível avançado de conhecimento científico (HÖLLDOBLER e WILSON, 1990; FERNANDEZ, 2003). Na Austrália, o uso de formigas como indicadores da reabilitação de áreas degradadas pelas atividades de mineração segue protocolos específicos, determinados após vários anos de estudos (MAJER et al., 1984; HOFFMAN et al., 2000; ANDERSEN et al., 2002). No Brasil, alguns estudos foram realizados na década de 90 (MAJER 1992/1996), mas a técnica permanece pouco explorada.

As formigas vêm sendo consideradas como um dos principais componentes biológicos de ambientes estruturalmente complexos como as florestas (FITTKAU e KLINGE, 1973). Muitas espécies são detritívoras e/ou predadoras de outros insetos, contribuindo para a reciclagem dos nutrientes e influenciando a dinâmica

1. Acadêmico do Curso de Engenharia Florestal, Instituto de Florestas, Universidade Federal Rural do Rio de Janeiro, Estrada Rio-São Paulo, BR 465, km 7, CEP 23851-970, Seropédica (RJ).

2. Engenheiro Agrônomo, Professor Adjunto, Departamento de Ciências Ambientais, Instituto de Florestas, Universidade Federal Rural do Rio de Janeiro, Estrada Rio-São Paulo, BR 465, km 7, CEP 23851-970, Seropédica (RJ).jarbas@ufrrj.br

3. Engenheiro Florestal, Professor Associado, Departamento de Ciências Ambientais, Instituto de Florestas, Universidade Federal Rural do Rio de Janeiro, Estrada Rio-São Paulo, BR 465, km 7, CEP 23851-970, Seropédica (RJ).

4. Biólogo, Professor Associado, Departamento de Biologia Animal, Instituto de Biologia, Universidade Federal Rural do Rio de Janeiro, Estrada Rio-São Paulo, BR 465, km 7, CEP 23851-970, Seropédica (RJ). Bolsista do CNPq.

Recebido para publicação em 28/10/2005 e aceito em 20/04/2007. 
populacional de insetos herbívoros (HÖLLDOBLER e WILSON, 1990). Vários estudos têm buscado compreender os impactos ocasionados pelas constantes simplificações dos ecossistemas naturais através de análise das comunidades de formigas (MAJER, 1996).

Diversos autores têm demonstrado a existência de correlação significativa entre características estruturais dos habitats e padrões de comunidades de formigas (ANDERSEN e MAJER, 2004). Uma abordagem para a utilização de formigas como ferramenta de monitoramento ambiental é a comparação das amostras tomadas em diferentes ambientes segundo a riqueza, diversidade e eqüitabilidade na distribuição de abundância das espécies encontradas (MAJER, 1983). Uma outra abordagem é a comparação das amostras utilizando os grupos funcionais de formigas propostos por Andersen (1990). O presente trabalho investigou a estrutura da comunidade de formigas como indicadora do sucesso de manejos para a reabilitação de uma área degradada por atividade mineradora na Bacia de Sepetiba, domínio Mata Atlântica, no Estado do Rio de Janeiro.

\section{MATERIAL E MÉTODO}

\section{Caracterização do local do estudo}

O trabalho foi desenvolvido em uma área com 10,81 ha no Distrito da Ilha da Madeira, Município de

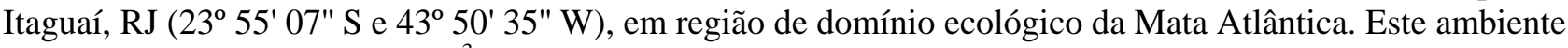
sofreu remoção de $1.400 .000 \mathrm{~m}^{3}$ de substrato (decapeamento médio de 13 metros) em 1979 para construção do Porto de Sepetiba, sendo posteriormente abandonado sem receber qualquer tratamento conservacionista (VALCARCEL e D`ALTÉRIO, 1998). Em 1994, foi realizada uma reabilitação do local envolvendo o uso de medidas físicas, biológicas e físico-biológicas. Diferentes combinações de espécies vegetais foram utilizadas como tentativa para a reabilitação dos processos ecológicos do ecossistema. Alguns tratamentos em parcelas, com tamanho médio de $300 \mathrm{~m}^{2}$, foram estabelecidos com diferentes combinações de espécies vegetais, que variavam em número e proporção de mudas das espécies arbóreas utilizadas, com apenas nativas (MB-5), nativas e exóticas (MB-2 e MB-4), apenas exóticas (MB-1) e também uma área testemunha, sem qualquer tipo de intervenção desde 1980. O tratamento MB-1 foi revegetado com $100 \%$ de Acacia auriculiformis A. Cunn. ex Benth; o MB-2 com 25\% de Acacia auriculiformis, 20\% com Leucaena leucocephala (Lam.) de Wit, 15\% com Piptadenia gonoacantha Mart. J.F. Macbr., 13\% com Caesalpinia ferrea Mart., 7\% com Albizia lebbech (L.) Benth., 10\% com Mimosa caesalpiniaefolia Benth., 5\% com Psidium guajava L. e 5\% com Tabebuia umbellata (Sond.) Sandwith; o MB-4 com 22\% de Acacia auriculiformis, 20\% com Clitoria fairchildiana R.A. Howard, 19\% com Inga laurina (Sw.) Willd., 16\% com Acacia mangium Willd., 11\% com Psidium guajava e 10\% com Piptadenia gonoacantha; e o MB-5 com $21 \%$ de Mimosa caesalpiniaefolia, 17\% com Clitoria fairchildiana, 15\% com Inga laurina, 15\% com Leucaena leucocephala, 13,5\% com Mimosa bimucronata (DC.) Kuntze, 7,5\% com Cecropia pachystachya Trécul., 6\% com Schinus terebinthifolius Raddi e 5\% com de Piptadenia gonoacantha (VALCARCEL e D’ALTÉRIO, 1998).

\section{Coleta de dados}

A coleta de formigas foi realizada nas parcelas descritas, utilizando-se quatro diferentes técnicas. A primeira técnica foi a coleta de colônias em galhos secos da serapilheira (galhos com 0,3 a $5 \mathrm{~cm}$ de diâmetro). O método adotado foi o de parcelas de $1 \mathrm{~m}^{2}$ (10 unidades) distantes $10 \mathrm{~m}$ entre si, totalizando 40 $\mathrm{m}^{2}$ de serapilheira analisada nos quatro tratamentos. Na área testemunha, devido à pequena produção de serapilheira, foram coletados todos os galhos no chão da parcela. Os galhos foram armazenados em sacos plásticos, etiquetados e levados ao laboratório para coleta e identificação das formigas. Uma segunda técnica utilizada foi a coleta manual de formigas, com auxílio de pinça entomológica, sobre o tronco das árvores. O método empregado foi a coleta em 10 árvores por tratamento, escolhidas aleatoriamente, durante 5 minutos/árvore. Esta técnica não foi empregada na área testemunha devido à inexistência de árvores. A terceira técnica utilizada foi a coleta manual na serapilheira com uso de iscas. O método utilizado foi o de espalhar 10 iscas por parcela, que consistia em uma pequena quantidade de sardinha conservada em óleo (uma colher de chá) sobre um papel branco, distantes 5 metros entre si. Após uma hora, as formigas atraídas para as iscas foram coletadas. A quarta técnica utilizada foi a coleta em armadilhas de solo do tipo pitfall. Foram utilizadas 10 armadilhas por parcela, distantes $5 \mathrm{~m}$ entre si, totalizando 50 armadilhas. As armadilhas utilizadas tinham $9 \mathrm{~cm}$ de diâmetro por $16 \mathrm{~cm}$ de altura e foram preenchidas com $100 \mathrm{ml}$ de solução de 
formalina (3\%). Elas permaneceram ativas no campo por 48 horas, após o qual o material coletado foi recolhido e levado ao laboratório para triagem e identificação das formigas. As espécies de formigas coletadas no estudo foram montadas em via seca e depositadas na Coleção Entomológica "Costa Lima", IB/UFRRJ.

\section{Análise dos dados}

A análise dos dados foi realizada utilizando-se medidas de similaridade de Sorensen e análise de agrupamento, por distância euclidiana, através do método de média (Average) (MAGURRAN, 1988). Para a análise de agupamento, foram utilizadas matrizes de presença e ausência das espécies de formigas para o total de técnicas de coleta e em separado para a técnica de armadilha de solo e coleta manual com isca. A separação das espécies em grupos funcionais seguiu o modelo proposto por Andersen (1990).

\section{RESULTADOS E DISCUSSÃO}

Obteve-se um total de 30 espécies de formigas distribuídas em 7 subfamílias na área de estudo. No geral, a riqueza em espécies foi maior no tratamento MB5 (25 espécies), que foi implantado com espécies arbóreas nativas, e menor na testemunha (14 espécies), que não recebeu intervenção. Os tratamentos MB4, MB1 e MB2 apresentaram, respectivamente, 77\% (23), 66\% (20) e 63\% (19) das espécies de formigas encontradas no estudo. O tratamento MB5 também apresentou maior riqueza em espécies do que a testemunha para cada uma das técnicas analisadas individualmente (Tabela 1).

A diferença verificada entre a área testemunha e o tratamento com espécies nativas (MB5) seguiu o padrão observado por Andersen e Majer (2004), sendo as áreas revegetadas bastante diferentes da área sem tratamento conservacionista em relação à comunidade de formigas. Neste estudo, a composição em espécies de formigas apresentou maior similaridade entre os tratamentos MB2/MB4 e MB5/MB4 (40,5\% e 39,6\%, respectivamente, pelo índice de Sorensen). A área testemunha e o MB5 apresentaram o menor índice de similaridade de Sorensen (28,2\%). A análise de agrupamento, considerando todas as técnicas em conjunto, revelou uma separação nítida das áreas, principalmente entre o tratamento MB5 e os demais (Figura 1a). O padrão de agrupamento das áreas não se alterou muito quando a análise foi realizada em separado para as técnicas de armadilhas pitfall (Figura 1b) e iscas (Figura 1c).

A distribuição das espécies, segundo os grupos funcionais propostos por Andersen (1990), revelou pouca diferença entre as áreas. Os efeitos de stress e perturbação na composição dos grupos funcionais em um ambiente permanecem pouco documentados para regiões de florestas tropicais úmidas (ANDERSEN, 2000). Neste estudo, as principais diferenças foram a presença de um grupo funcional a mais nos tratamentos MB5 e MB4, o grupo "Oportunistas", e a maior proporção de espécies classificadas em "Camponotinae subordinadas” na área testemunha e no MB1 (Figura 2).

TABELA 1: Espécies de formigas coletadas com diferentes técnicas em área testemunha e tratamentos de reabilitação em área de mineração na Ilha da Madeira, Itaguaí-RJ. Legenda: I = isca; C = tronco; $\mathrm{G}=$ galhos; $\mathrm{P}=$ armadilhas pitfall.

TABLE 1: Ant species sampled with different techniques in control plot and treatments for rehabilitation of a mined area at Ilha da Madeira, Itaguaí-RJ. Legend: $\mathrm{I}=$ bait; $\mathrm{C}=$ tree trunk; $\mathrm{G}=$ twigs; $\mathrm{P}=$ pitfall traps.

\begin{tabular}{|c|c|c|c|c|c|}
\hline Subfamílias & & stem & Inha & & ME \\
\hline & I & C & $\mathrm{P}$ & I & ᄃ \\
\hline
\end{tabular}

\section{Ecitoninae}

Labidus sp.

$\mathrm{X}$

Ectatomminae

Ectatomma brunneum Fr. Smith, 1858

Formicinae

Brachymyrmex sp.1

X

$\mathrm{X}$

$\mathrm{X}$

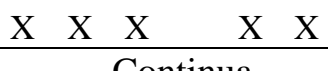

Continua ... 
TABELA 1: Continuação ...

TABLE 1: Continued ...

\begin{tabular}{|c|c|c|c|c|c|c|c|c|c|c|c|c|c|c|c|c|c|c|c|c|}
\hline \multirow[t]{2}{*}{ Subfamílias } & \multicolumn{4}{|c|}{ Testemunha } & \multicolumn{4}{|c|}{ MB1 } & \multicolumn{4}{|c|}{ MB2 } & \multicolumn{4}{|c|}{ MB4 } & \multicolumn{4}{|c|}{ MB5 } \\
\hline & $\mathrm{I}$ & \begin{tabular}{|l|l|l}
$\mathrm{C}$ & $\mathrm{C}$ \\
\end{tabular} & \begin{tabular}{l|l}
$G$ & $P$ \\
\end{tabular} & $\mathrm{P}$ & I & 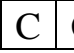 & G & $\mathrm{P}$ & I & $\mathrm{C}$ & $\mathrm{G}$ & $\mathrm{P}$ & I & $\mathrm{C}$ & $\mathrm{G}$ & $\mathrm{P}$ & I & \begin{tabular}{l|l}
$\mathrm{C}$ & $\mathrm{C}$ \\
\end{tabular} & $\mathrm{G}$ & $\mathrm{P}$ \\
\hline Brachymyrmex sp.2 & & & & $\mathrm{X}$ & & & & $\mathrm{X}$ & & & & $\mathrm{X}$ & & & & $\mathrm{X}$ & & & & $\mathrm{X}$ \\
\hline Camponotus crassus Mayr, 1862 & & & & $\mathrm{X}$ & $\mathrm{X}$ & & $\mathrm{X}$ & $\mathrm{X}$ & $\mathrm{X}$ & $\mathrm{X}$ & & $\mathrm{X}$ & $\mathrm{X}$ & $\mathrm{X}$ & $\mathrm{X}$ & $\mathrm{X}$ & $\mathrm{X}$ & $\mathrm{X}$ & $\mathrm{X}$ & $\mathrm{X}$ \\
\hline Camponotus rufipes (Fabricius, 1775) & $\mathrm{X}$ & & & $\mathrm{X}$ & $\mathrm{X}$ & & & $\mathrm{X}$ & $\mathrm{X}$ & $\mathrm{X}$ & & $\mathrm{X}$ & $\mathrm{X}$ & & & $\mathrm{X}$ & & & & \\
\hline Camponotus sp. & & & & $\mathrm{X}$ & & & & $\mathrm{X}$ & & & & $\mathrm{X}$ & & & & $\mathrm{X}$ & & & & $\mathrm{X}$ \\
\hline Paratrechina sp. & & & & & & & & & & & & & & $\mathrm{X}$ & & & & $\mathrm{X}$ & $\mathrm{X}$ & \\
\hline \multicolumn{21}{|l|}{ Myrmicinae } \\
\hline Acromyrmex sp. & & & & $\mathrm{X}$ & & & & $X$ & & & & & & & & & & & & \\
\hline Atta sp. & & & & $\mathrm{X}$ & & & & $\mathrm{X}$ & & & & $\mathrm{X}$ & & & & $\mathrm{X}$ & & $\mathrm{X}$ & & $\mathrm{X}$ \\
\hline Cephalotes pusillus (Klug, 1824) & $\mathrm{X}$ & & & & $\mathrm{X}$ & $\mathrm{X}$ & & $\mathrm{X}$ & $\mathrm{X}$ & $\mathrm{X}$ & & & $\mathrm{X}$ & $\mathrm{X}$ & & $\mathrm{X}$ & & & & \\
\hline Crematogaster sp. & $\mathrm{X}$ & & & $\mathrm{X}$ & $\mathrm{X}$ & & & & & & & $\mathrm{X}$ & & $\mathrm{X}$ & & $\mathrm{X}$ & $\mathrm{X}$ & $\mathrm{X}$ & & \\
\hline Hylomyrma sp. & & & & & & & & & & & & & & & & & $\mathrm{X}$ & & & \\
\hline Mycetarotes parallelus (Emery, 1905) & & & & & & & & & & & & & & & & & & & & $\mathrm{X}$ \\
\hline Pheidole sp.1 & & & & & & $\mathrm{X}$ & & & $\mathrm{X}$ & & & & & $\mathrm{X}$ & & & & $\mathrm{X}$ & $\mathrm{X}$ & \\
\hline Pheidole sp.2 & & & & & $\mathrm{X}$ & $\mathrm{X}$ & & & & & & & $\mathrm{X}$ & $\mathrm{X}$ & & $\mathrm{X}$ & $\mathrm{X}$ & $\mathrm{X}$ & $\mathrm{X}$ & $\mathrm{X}$ \\
\hline Pheidole sp.3 & & & & & $\mathrm{X}$ & & & $\mathrm{X}$ & $\mathrm{X}$ & & & $\mathrm{X}$ & $\mathrm{X}$ & & & & $\mathrm{X}$ & & & \\
\hline Pheidole sp.4 & & & & & $\mathrm{X}$ & & & $\mathrm{X}$ & $\mathrm{X}$ & & & $\mathrm{X}$ & $\mathrm{X}$ & & & $\mathrm{X}$ & $\mathrm{X}$ & & & \\
\hline Pheidole sp.5 & $\mathrm{X}$ & & & & & & & $\mathrm{X}$ & $\mathrm{X}$ & & & & & & & $\mathrm{X}$ & $\mathrm{X}$ & $\mathrm{X}$ & & \\
\hline Solenopsis sp.1 & & & & & $\mathrm{X}$ & & & $\mathrm{X}$ & & & & $\mathrm{X}$ & & & & $\mathrm{X}$ & $\mathrm{X}$ & & & \\
\hline Solenopsis sp.2 & & & & & $\mathrm{X}$ & & & & & & & & & & & & & & & $\mathrm{X}$ \\
\hline Strumigenys sp. & & & & & & & & $\mathrm{X}$ & & & & & & & & $\mathrm{X}$ & & & & \\
\hline Trachymyrmex sp. & & & & & & & & & & & & $\mathrm{X}$ & & & & $\mathrm{X}$ & & & & $\mathrm{X}$ \\
\hline Wasmannia auropunctata (Roger, 1863) & $\mathrm{X}$ & & & $\mathrm{X}$ & $\mathrm{X}$ & & & $\mathrm{X}$ & $\mathrm{X}$ & & $\mathrm{X}$ & $\mathrm{X}$ & & & $\mathrm{X}$ & $\mathrm{X}$ & $\mathrm{X}$ & & $\mathrm{X}$ & $\mathrm{X}$ \\
\hline \multicolumn{21}{|l|}{ Ponerinae } \\
\hline Pachycondyla striata Fr. Smith, 1858 & & & & $\mathrm{X}$ & $\mathrm{X}$ & & & $\mathrm{X}$ & & & & $\mathrm{X}$ & & & & $\mathrm{X}$ & $\mathrm{X}$ & & & $\mathrm{X}$ \\
\hline \multicolumn{21}{|l|}{ Pseudomyrmecinae } \\
\hline Pseudomyrmex sp.1 & & & & & & $\mathrm{X}$ & & $\mathrm{X}$ & & $\mathrm{X}$ & & $\mathrm{X}$ & & $\mathrm{X}$ & & $\mathrm{X}$ & & $\mathrm{X}$ & & $\mathrm{X}$ \\
\hline Pseudomyrmex sp.2 & & & & & & & & & & $\mathrm{X}$ & & & & & & & & $\mathrm{X}$ & & \\
\hline Pseudomyrmex sp.3 & $\mathrm{X}$ & & & $\mathrm{X}$ & & & & & & & & & & $\mathrm{X}$ & & & $\mathrm{X}$ & $\mathrm{X}$ & & \\
\hline Pseudomyrmex sp.4 & & & $\mathrm{X} \quad \mathrm{X}$ & $\mathrm{X}$ & & & & & $\mathrm{X}$ & & & & & & $\mathrm{X}$ & & $\mathrm{X}$ & & & \\
\hline Pseudomyrmex sp.5 & & & & & & & & & & & & $\mathrm{X}$ & & & & & & & & $\mathrm{X}$ \\
\hline Total por técnica/ambiente & 7 & 0 & 11 & 11 & 11 & 4 & 1 & 16 & 9 & 5 & 1 & 14 & 7 & 8 & 4 & 17 & 14 & 10 & 6 & 14 \\
\hline Total por ambiente & & 14 & & & & 20 & & & & & 19 & & & 2 & 3 & & & 25 & & \\
\hline
\end{tabular}




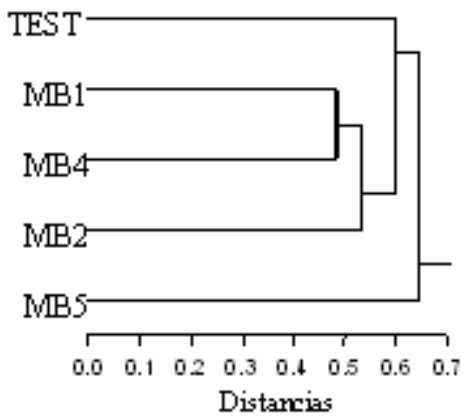

(a)

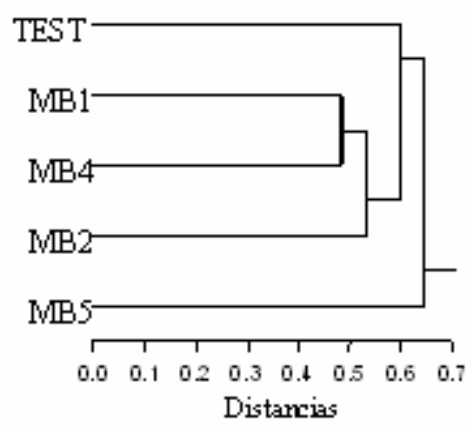

(b)

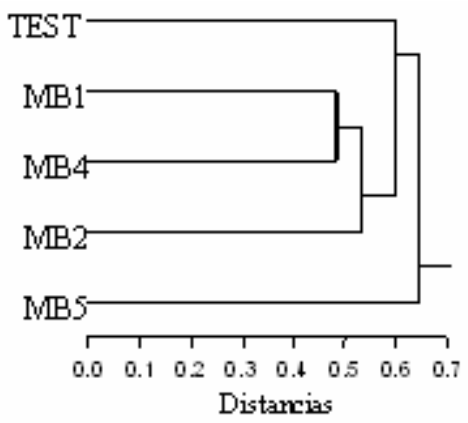

(c)

FIGURA 1: Análise de agrupamento das parcelas, com matriz de presença e ausência das espécies de formigas, utilizando distância euclidiana, pelo método da média (average). a - todas as técnicas em conjunto. b - apenas técnica de armadilhas pitfall. c - apenas técnica de coleta manual em isca. TEST - parcela testemunha; MB1, MB2, MB4 e MB5 - tratamentos de medidas biológicas. Para detalhes sobre os tratamentos ver texto.

FIGURE 1: Cluster analysis utilizing presence/absence matrix for ant species (euclidian distance, average method). a - pooled techniques. b - pitfall traps. c - hand collected ants on sardine baits. TEST - control plot; MB1, MB2, MB4 and MB5 - revegetated plots. See text for details. 


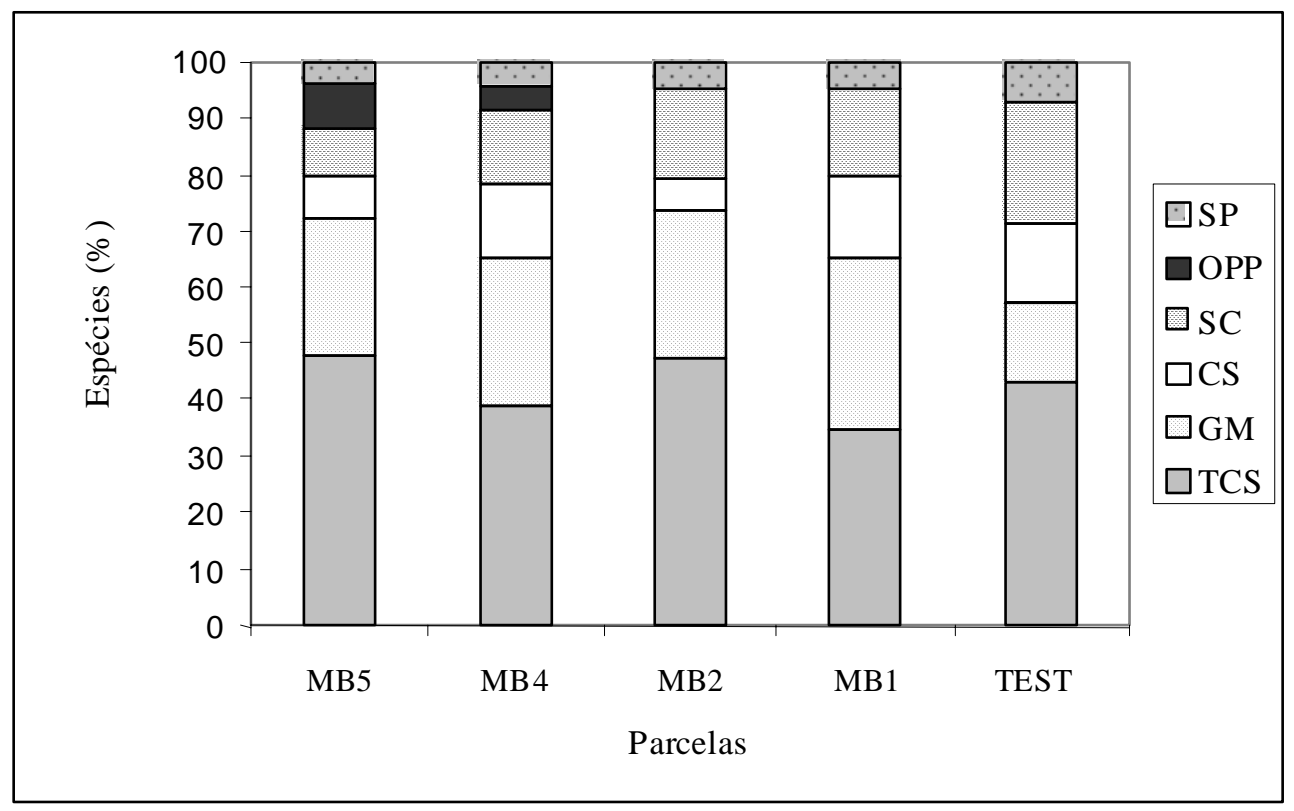

FIGURA 2: Distribuição percentual das espécies de formigas em grupos funcionais nas diferentes parcelas amostradas. $\mathrm{SP}=$ predadores especialistas; $\mathrm{OPP}=$ oportunistas; $\mathrm{SC}=$ Camponotinae subordinadas; $\mathrm{CS}$ = espécies crípticas; GM = Myrmicinae generalizados; TCS = especialistas de climas tropicais (segundo Andersen, 1990).

FIGURE 2: Ant species distribution among functional groups in the plots. SP = specialist predators; OPP = opportunists; SC = subordinate Camponotini; $\mathrm{CS}=$ cryptic species; $\mathrm{GM}=$ generalized Myrmicinae; TCS = tropical climate specialists (following Andersen, 1990).

Silvestre (2000) considerou as espécies de Camponotus como oportunistas e generalistas em termos de dieta e local para nidificação. Muitas espécies de Camponotus são tipicamente encontradas em ambientes degradados com alta incidência de luz solar (LONGINO, 2005). Em toda a área reabilitada, as espécies Camponotus crassus e Camponotus rufipes são muito comuns, estando a diferença no fato de que as parcelas reabilitadas com uma maior variedade de espécies arbóreas possuíam uma fauna mais rica em espécies, deixando um peso maior para as espécies de Camponotus na área testemunha.

A maior riqueza de formigas e uma fauna diferenciada nas parcelas amostradas podem estar relacionadas com a maior complexidade estrutural da serapilheira, advinda da estratégia de reabilitação que utilizou maior número de pioneiras nativas nos tratamentos MB5 e MB4 (VALCARCEL e DÁLTÉRIO, 1998). Uma parcela dos galhos presentes nos tratamentos MB5 e MB4 possuíam características apropriadas para a utilização por formigas - i.e. moles, ocos ou com orifícios (CARVALHO e VASCONCELOS, 2002). Disso resultou um maior número de espécies de formigas encontradas nidificando em galhos nesses tratamentos (6 e 4 espécies, respectivamente) do que na testemunha (1 espécie) ou nos outros tratamentos (1 espécie). A simples oferta de galhos secos de diferentes espécies arbóreas, utilizadas nesses tratamentos, em comparação com a menor variedade de galhos na área testemunha ou o predomínio de apenas alguns tipos de galhos nos tratamentos MB1 e MB2 pode também explicar as diferenças na riqueza de espécies de formigas (ARMBRETCH et al., 2004).

Estes resultados confirmam diversos autores que sugerem que a riqueza de espécies de formigas esteja correlacionada com a complexidade estrutural do ambiente (VASCONCELOS, 1998; ARMBRETCH et al., 2004). O aumento da diversidade em um determinado nível cria condições para o aumento em outros níveis (ARMBRECHT et al., 2004). Segundo Neves (2004), o tratamento MB5 tem maior diversificação estrutural dos componentes arbóreos e aproxima-se estrutural e fisionomicamente de ambientes sucessionais mais avançados de Mata Atlântica, além de apresentar maior decomposição da serapilheira que os demais tratamentos. Ambientes degradados ou com baixa diversificação vegetal (homogêneos) apresentam limitações à presença de organismos, devido à falta de recursos proporcionados por estes ambientes. Assim, nessas áreas, as comunidades de formigas podem se apresentar com baixa diversidade de espécies e grupos 
funcionais.

\section{CONCLUSÕES}

As comunidades de formigas são mais ricas em espécies em ambientes reabilitados do que em ambientes degradados sem tratamento conservacionista. A composição em espécies também varia segundo a estrutura da vegetação. Ambientes reabilitados através da implantação de espécies arbóreas nativas apresentam fauna diferenciada em relação aos ambientes sem intervenção ou reabilitados com espécies arbóreas exóticas. A comunidade de formigas investigada demonstrou ser sensível às variações no ambiente, sofrendo influência da estrutura vegetal. Através do monitoramento das comunidades de formigas em áreas reabilitadas é possível avaliar as metodologias e a eficácia das técnicas de revegetação quanto à manutenção da diversidade local e, conseqüentemente, a autosustentabilidade destes ambientes.

\section{AGRADECIMENTOS}

Os autores agradecem a dois revisores anônimos, pelas sugestões em uma versão prévia do manuscrito, e à Sepetiba Engenharia e Comércio Ltda, pela oportunidade para trabalhar na área da empresa através do convênio firmado com o Laboratório de Manejo de Bacias Hidrográficas (UFRRJ).

\section{REFERÊNCIAS BIBLIOGRÁFICAS}

ANDERSEN, A.N. The use of ant communities to evaluate changes in Australian terrestrial ecosystems: a review and a recipe. Proceedings of the Ecological Society of Australia, Canberra, v. 16, p. 347-357, 1990.

ANDERSEN, A.N. Global ecology of rainforest ants: functional groups in relation to environmental stress and disturbance. In: AGOSTI, D.; MAJER, J.D.; ALONSO, L.E. AND SCHULTZ, T., eds. Ants: standard methods for measuring and monitoring biodiversity. Washington: Smithsonian Institutional Press, 2000, p.25-34.

ANDERSEN, A.N.; MAJER, J.D. Ants show the way Down Under: invertebrates as bioindicators in land management. Frontiers in Ecology and the Environment, Silver Spring, v. 2, p. 291-298, 2004.

ANDERSEN, A.N.; HOFFMANN, B.D.; MÜLLER, W. J. et al. Using ants as bioindicators in land mangement: simplifying assessment of ant community responses. Journal of Applied Ecology, Oxford, v. 39, p.8-17, 2002.

ARMBRECHT, I.; PERFECTO, I.; VANDERMEER, J. Enigmatic biodiversity correlations: ant diversity responds to diverse resources. Science, Washington, v. 304, p. 284-286, 2004.

CARVALHO, K.; VASCONCELOS, H. Comunidade de formigas que nidificam em pequenos galhos da serrapilheira em floresta da Amazônia Central, Brasil. Revista Brasileira de Entomologia, Curitiba, v. 46, p.115-121, 2002.

FERNANDEZ, F. Introducción a las hormigas de la región Neotropical. Bogotá: Acta Nocturna, 2003. 418p.

FITTKAU, E. J.; KLINGE, H. On biomass and trophic structure of the Central Amazonian rain forest Ecosystem. Biotropica, Oxford, v. 5, p. 2-14, 1973.

HOFFMANN B.D.; GRIFFITHS, A.D.; ANDERSEN, A.N. Responses of ant communities to dry sulfur deposit form mining emissions in the semi-arid tropical Australia, with implications for the use of functional groups. Austral Ecology, Oxford, v. 25, p.653-663, 2000.

HÖLLDOBLER, B.; WILSON, E.O. The Ants. Cambridge: Harvard University Press, 1990. 738p.

LONGINO, J.T. Ants of Costa Rica. Disponível em: <http://www.evergreen.edu/ants/antsofcostarica. html> Acesso em: 01 ju. 2005.

MAGURRAN, A.E. Ecological diversity and its measurement. London: Croom Helm, 1988. 178p.

MAJER, J.D. Ants: bioindicators of Minesite Rehabilitation, land use, and land conservation. Enviromental Managment, New York, v.7, p.375-383, 1983.

MAJER, J.D. Ant recolonization of rehabilitated bauxite mines of Poços de Caldas, Brazil. Journal of Tropical Ecology, Winchelsea, v.8, p.97-108, 1992.

MAJER, J.D. Ant recolonization of rehabilitated bauxite mines at Trombetas, Pará, Brazil. Journal of Tropical Ecology, Winchelsea, v.12, p.257-273, 1996.

MAJER, J.D.; DAY, J.E.; KABAY, E.D. et al. Recolonization by ants in bauxite mines rehabilitated by a number of different methods. Journal of Applied Ecology, Oxford, v.21, p.355-375, 1984.

NEVES, L.G. Eficiência conservacionista de medidas biológicas em áreas degradadas no domínio ecológico da Mata Atlântica. 2004. 111f. Dissertação (Mestrado em Ciências Ambientais e Florestais) - Instituto de Florestas, 
Universidade Federal Rural do Rio de Janeiro, Seropédica.

ROSENBERG, D.M.; DANKS, H.V.; LEHMKUHL, D.M. Importance of insects in environmetal impact assessment. Environmental Management, New York, v.10, p.773-783, 1986.

SILVESTRE, R. Estrutura de comunidades de formigas do cerrado. 2000. 216f. Tese (Doutorado em CiênciasEntomologia) - FFCL, USP, Ribeirão Preto.

VALCARCEL, R.; D`ALTÉRIO, C. F. Medidas físico-biologicas de recuperação de áreas degradadas: avaliação das modificações edáficas e fitossociológicas. Floresta \& Ambiente, Seropédica, v.5, p.68-88, 1998.

VASCONCELOS, H.L. Respostas das formigas à fragmentação florestal. Série Técnica IPEF, Piracicaba, v.12, p.95-98, 1998.

WARD, D.F.; NEW, T.R.; YEN, A.L. Effects of pitfall trap spacing on the abundance, richness and composition of invertebrate catches. Journal of Insect Conservation, Netherlands, v.5, p.47-53, 2001. 